\title{
Cubic octanuclear aluminum fluoride phosphonate
}

Jan Kratochvil, ${ }^{\dagger}$ Marek Necas, $^{\dagger}$ Vaclav Petricek, ${ }^{\ddagger}$ and Jiri Pinkas ${ }^{\dagger,{ }^{*}}$

${ }^{\dagger}$ Department of Inorganic Chemistry, Masaryk University, Kotlarska 2, CZ-61137 Brno, Czech Republic

${ }^{\ddagger}$ Institute of Physics, Academy of Sciences of the Czech Republic, Na Slovance 2, CZ18221 Praha, Czech Republic 
Fig 1. Packing diagram of 1 along the crystallographic $a$-axis. Only one of the two disordered components are shown for each position. Please note that the molecules of water fill the space between the molecules of cubic aluminium fluoride phosphonates, they are not enclosed within.

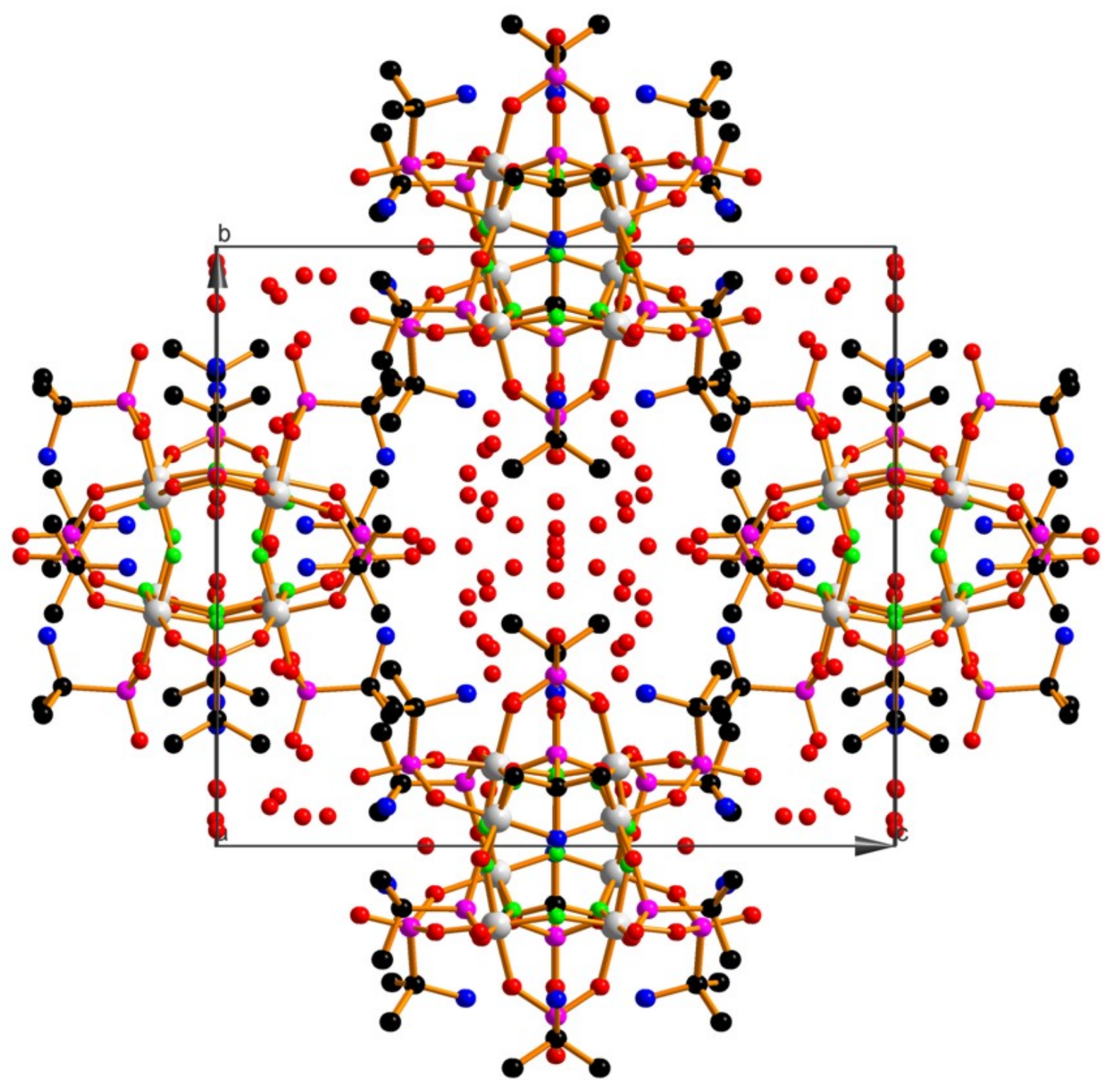


Fig 2. Packing diagram of 1 along the crystallographic $c$-axis. Only one of the two disordered components are shown for each position. Please note that the molecules of water fill the space between the molecules of cubic aluminium fluoride phosphonates, they are not enclosed within.

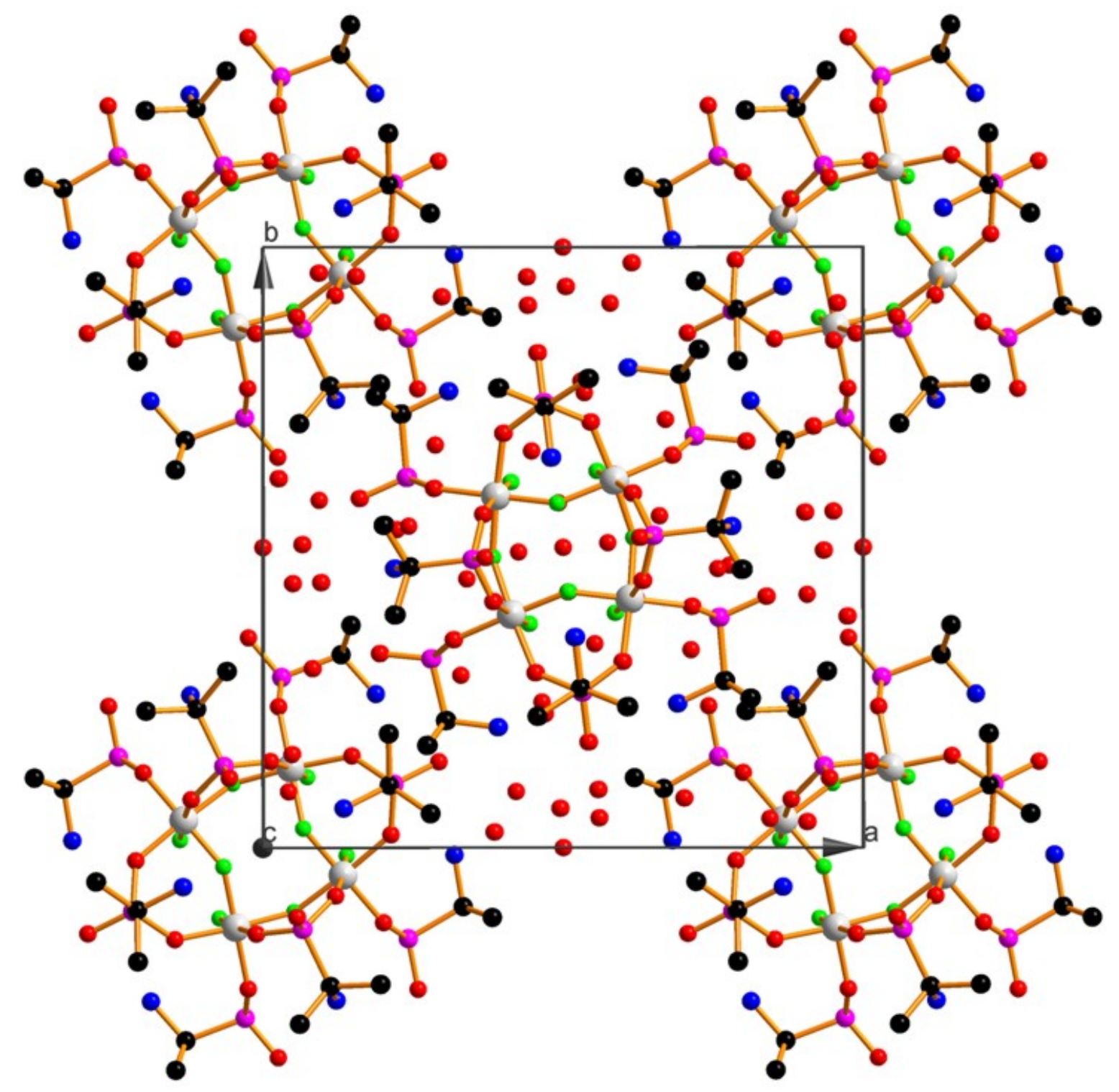

